\title{
Abnormal left ventricular wall movement during early systole in patients with angina pectoris
}

\author{
D. G. GIBSON, J. H. DORAN, 1 T. A. TRAILL, AND D. J. BROWN \\ From the Brompton Hospital, London
}

SUMMARY Left ventricular cineangiograms of 105 patients with chest pain were digitised frame by frame and local endocardial movement displayed in order to study events during isovolumic contraction. Of these patients, 90 had coronary artery disease and the remainder were considered normal. In normal subjects, there was a 6 per cent reduction in cavity area before the onset of aortic valve opening, caused by inward movement of endocardium on the upper part of the anterior wall, so that the cavity became less circular. In 34 patients with coronary artery disease, abnormalities of isovolumic contraction were present. These consisted of: (1) segmental outward movement of $2 \mathrm{~mm}$ or more (mean $4 \pm 2 \mathrm{~mm}$ ) in 23, and (2) premature inward movement along the inferior wall in a further 11 . Of the former 23 patients, overall amplitude of wall movement during ejection was greater than 60 per cent than in control regions of the same ventricle in 14 (type I), and less in 9 (type II), because of local disease. These abnormalities of isovolumic contraction were unrelated to the distribution of coronary artery lesions, but were more frequent in the presence of anterior $Q$ waves. Type I abnormalities also occurred in 3 additional patients with normal coronary arteriograms. All but 2 of the patients with type I abnormalities had additional disturbances of endocardial movement during isovolumic relaxation, affecting the same region of ventricle. Disturbances of endocardial movement during isovolumic contraction are thus relatively common in patients with angina pectoris, and may cause significant interference with left ventricular function.

Although regional abnormalities of left ventricular function occurring during ejection have been extensively studied in patients with ischaemic heart disease, those during isovolumic contraction have received less attention. Nevertheless, synchronous and rapid onset of contraction with little change in cavity shape before aortic valve opening appears essential for normal left ventricular function. Previous studies in man have identified changes in left ventricular volume (Karliner et al., 1971), and in transverse dimension, measured by angiography (Karliner et al., 1971), echocardiography (Venco et al., 1977), or movement of epicardial markers (McDonald, 1970). In the present study, we have used methods of displaying left ventriculograms, which allow local movement of endocardium around the cavity outline to be appreciated throughout the cardiac cycle in order to provide a more com-

${ }^{1}$ Present address: Medical Center Hospital, Odessa, Texas, U.S.A.

Received for publication 1 September 1977 prehensive account of events during isovolumic contraction and immediately after aortic valve opening. The nature and position of these disturbances have been correlated with coronary artery anatomy, the electrocardiogram, and subsequent wall movement during ejection and isovolumic relaxation in order to gain some idea as to their genesis and effects on overall left ventricular function.

\section{Methods}

One hundred and five patients were investigated for pain thought to be caused by ischaemic heart disease. Fifteen patients proved to have normal coronary artery anatomy, intracardiac pressures, and left ventricular volumes and ejection fractions, and are referred to as a 'normal' group. The remainder all had significant coronary artery disease, except that in 4, with severe and generalised left ventricular disease, coronary arteriography was not performed. 
Left ventriculograms were performed in all patients before coronary arteriography, using methods previously described (Gibson et al., 1976).

\section{ANALYSIS OF LEFT VENTRICULOGRAMS}

Left ventriculograms were digitised frame by frame, using a Prime 300 computer system and a Summagraphics digitiser. The information was stored on disc, and from it the following information was obtained:

(1) Superimposed plots of cavity outlines during systole and diastole (Gibson and Brown, 1975), which were used to assess overall amplitude of wall movement during the cardiac cycle. For simplicity, the cavity outline was considered to consist of only three regions, anterior, apical, and inferior, and in each region, movement was considered to be either normal or reduced in amplitude.

(2) Graphs showing cavity area, derived volume, perimeter, long axis, and shape index (4 (perimeter) ${ }^{2}$ Gibson and Brown, 1975), continuously throughout the cardiac cycle. On these graphs were superimposed the timing of: (a) the onset of aortic valve opening, determined from direct inspection of the angiogram, (b) minimum cavity area, and (c) mitral valve opening, taken as the time of first appearance of unopacified blood from the left atrium within the left ventricular cavity. From these graphs, the following quantities were determined: (1) ejection fraction, as (stroke volume)/(enddiastolic volume), (2) end-diastolic perimeter, and (3) any change in cavity area, long axis, or shape index between the time of maximum cavity area and aortic valve opening.

(3) Plots showing regional movement of left ventricular endocardium. The rationale and limitations of this method have been described elsewhere (Gibson et al., 1976). It depends on defining 40 equally spaced points on the end-diastolic cavity outline starting from the border of the aortic root adjacent to the mitral valve, and proceeding in an anticlockwise direction. From each, the nearest point on the end-systolic frame is sought, and along the line thus defined, wall position in each cine frame is measured and expressed in $\mathrm{mm}$ from its end-diastolic position. The resulting 40 plots of wall movement against time were displayed either stacked obliquely, with superimposed isochrones to represent the timing of successive cine frames (Fig. 1) or as a contour map (Fig. 2), where each contour represents inward movement of endocardium by $1 \mathrm{~mm}$ from end-diastolic position. On both types of display, the timing of aortic valve opening, minimum cavity area, and mitral valve opening were marked. Endocardial movement before aortic valve opening was analysed from these displays,

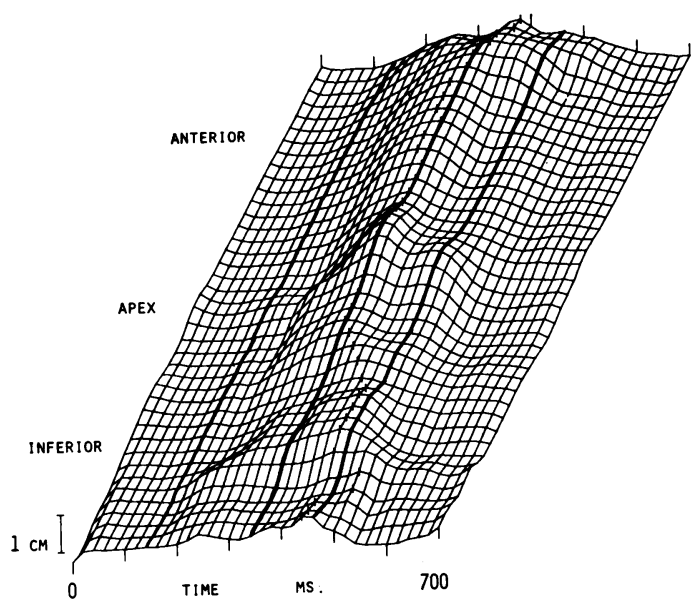

Fig. 1 Isometric plot of endocardial movement in a normal subject. Inward endocardial movement is shown by upward displacement of each plot of position against time. The diagonal lines represent the timing of successive cine frames, and the accentuated lines represent aortic valve opening ( $A V O)$, minimum cavity area, and mitral valve opening, from left to right.

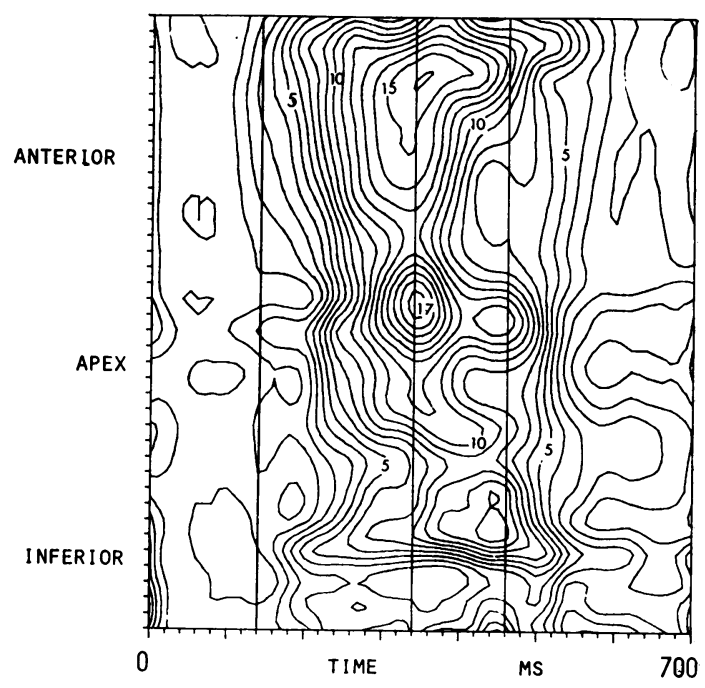

Fig. 2 Contour plot of the data of Fig. 1. Contour lines represent inward movement of endocardium by $1 \mathrm{~mm}$ from the position in the end-diastolic frame. As in Fig. 1, the three vertical lines represent the times of aortic valve opening, minimum cavity area, and mitral valve opening. Inward movement precedes $A V O$ in the high anterior region, but is delayed elsewhere. 
with particular reference to the presence and distribution of either abnormal outward or premature inward movement, defined with respect to the results in the normal patients (Results, Section 1). The extent of such abnormal movement around the cavity was estimated from the number of separate plots in which it appeared: since there were $\mathbf{4 0}$ around the whole outline, such estimates could be converted to $\mathrm{mm}$ knowing simultaneous cavity perimeter. From the isometric display the plot in which the extent of the disturbance to wall movement was greatest could be selected and studied separately, along with its rate of change of position and its exact site with respect to end-diastolic and end-systolic cavity outlines. From each patient a second plot was selected, usually from the anterior wall, in which movement was normal, to serve as a basis for comparison. From each of these plots measurements were made of (a) the greatest outward movement of endocardium, with its timing in relation to that of aortic valve opening; (b) the magnitude and timing of the peaks on the curve of rate of change of endocardial position with time; and (c) the duration of inward movement.

(4) In order to explore the clinical associations of abnormal wall movement before aortic valve opening the following additional information was available in each patient:

(a) The presence of anterior or inferior $Q$ waves on the electrocardiogram.

(b) Coronary artery anatomy, in terms of the three main vessels. Significant stenosis was con- sidered to be present when the lumen was narrowed by more than 70 per cent. No attempt was made to estimate collateral flow.

(c) The presence of abnormal left ventricular endocardial movement between the times of minimum cavity area and mitral valve opening (Gibson et al., 1976).

\section{Results}

\section{(A) NORMAL SUBJECTS}

A characteristic series of events occurred before aortic valve opening in 12 out of the 15 normal subjects. There was a reduction in cavity area by 3 to 14 per cent (mean $6 \%$ ) in all but one, associated with a change in cavity configuration, indicating that it had become less circular, shape index falling by 3 per cent (range 0 to $6 \%$ ). There was no significant change in long axis. Plots of regional endocardial movement were also characteristic, since in all 12 cases, the reduction in cavity area was the result of inward movement of the anterior wall adjoining the aortic root over a region 25 to $40 \mathrm{~mm}$ towards the apex, by a mean of $2 \pm 1 \mathrm{~mm}$ (mean $\pm 1 \mathrm{SD}$ ). This always preceded movement in the inferior region of the cavity, which never began until after the onset of aortic valve opening. The last region to show movement was the apex at a mean interval of $65 \pm 15$ ms after aortic valve opening (Fig. 2).

In 3 patients in the normal group, abnormal outward movement occurred at the apex, identical to that described in the next section as type I.

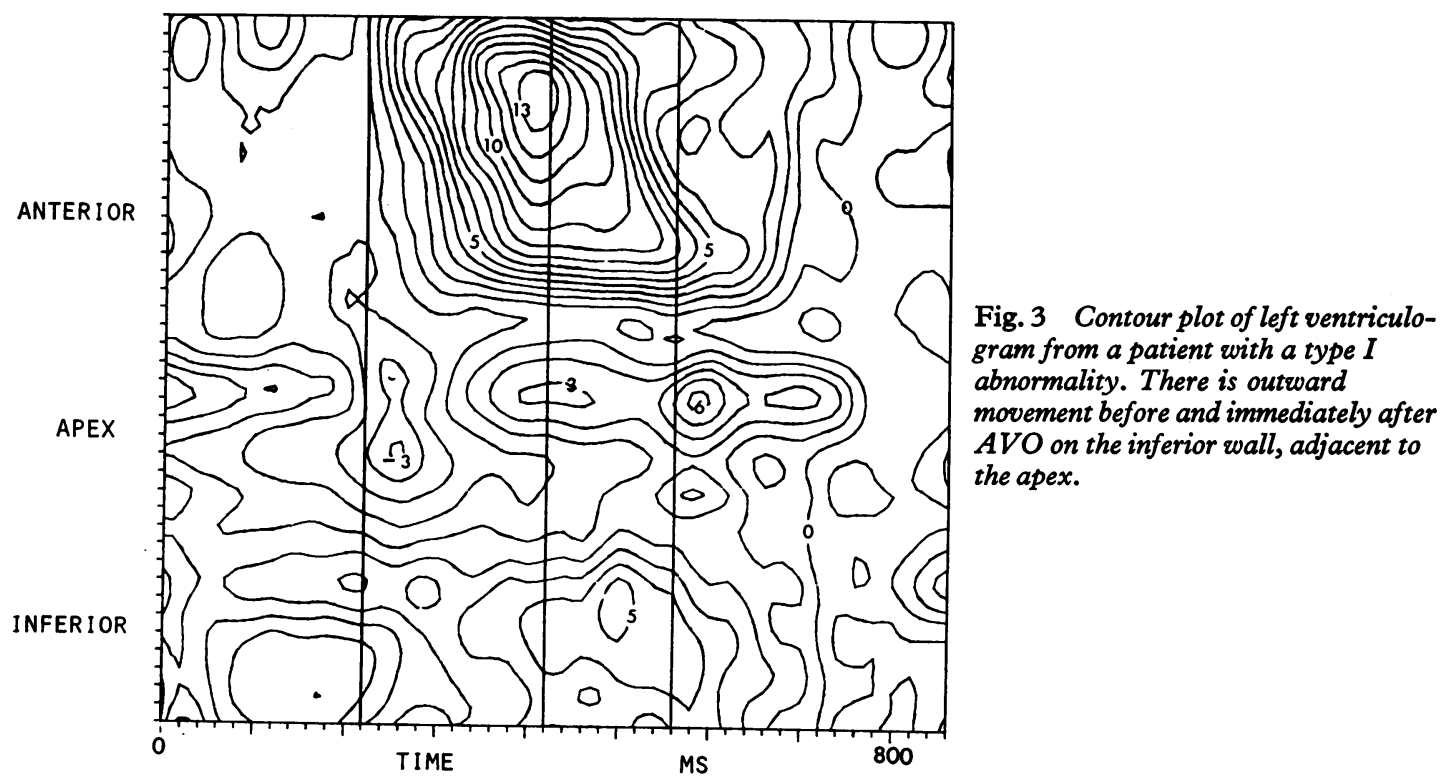




\section{(B) ISCHAEMIC HEART DISEASE}

On the basis of events occurring in normal subjects, a series of abnormalities has been defined in patients with ischaemic heart disease. In 23 patients, significant outward movement was apparent in one region of the cavity before aortic valve opening. Such regions were localised on isometric or contour displays, and they were further divided retrospectively into two groups, depending on whether overall amplitude of movement of the affected area was greater or less than 60 per cent that of the unaffected area of the same ventricle.

Type I (14 patients) (Figs. 3 to 5)

In this group, the amplitude of wall movement was greater than 60 per cent of that in the control regions, having a mean of $10 \pm 4 \mathrm{~mm}$, compared with control of $14 \pm 4 \mathrm{~mm}$. The mean amplitude of outward movement was $4 \mathrm{~mm}$ (range 2 to $8 \mathrm{~mm}$ ) and though starting before aortic valve opening, reached its greatest extent at 75 (range 50 to $100 \mathrm{~ms}$ ) afterwards (Fig. 5). The site of the plot showing maximum outward movement is shown in Fig. 6, and it is apparent that these cluster at the apex and along the adjoining region of the inferior wall. The extent around the cavity perimeter ranged from 20 to $45 \mathrm{~mm}$. The duration of inward movement was $290 \pm 50 \mathrm{~ms}$, compared with control of $270 \pm$ $60 \mathrm{~ms}$, values that were not significantly different. Peak rate of inward movement in affected areas was $9 \pm 5 \mathrm{~cm} / \mathrm{s}$, no different from that in control regions, $11 \pm 4 \mathrm{~cm} / \mathrm{s}$. The form of the plot of velocity against time was characteristic in that in affected areas it had two peaks, one early in systole

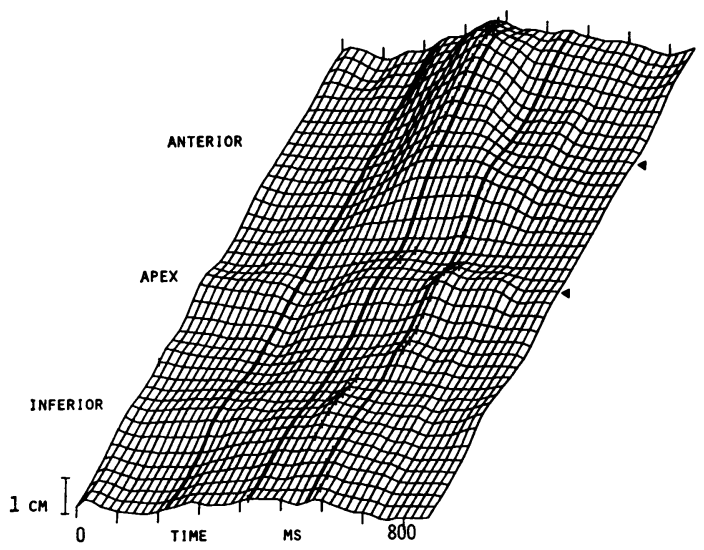

Fig. 4 Isometric plot of the data of Fig. 3. The positions of the two plots analysed in greater detail in Fig. 5 are shown by arrows.
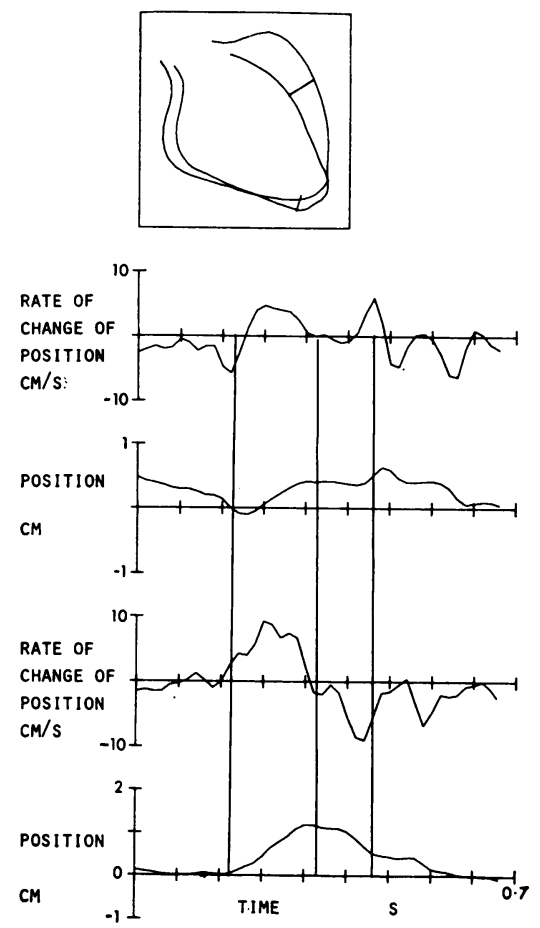

Fig. 5 Analysis of regional wall movement. The lower two plots show endocardial movement and its rate of change from a normal region and the upper two from the apical region showing a type I abnormality. The position of these plots with reference to end-systolic and end-diastolic cavity outlines are shown in the top panel. Vertical lines represent the times of $A V O$, minimum cavity area, and mitral valve opening. Abnormal outward movement before AVO and inward movement during isovolumic relaxation, reaching its maximum velocity at the time of mitral valve opening are apparent.

as the endocardium was moving back towards its end-diastolic position, and one during isovolumic relaxation immediately before mitral valve opening. This second peak in the velocity trace was associated with abnormal inward movement of endocardium in 10 out of 12 cases of 3 to $6 \mathrm{~mm}$ during isovolumic relaxation.

An identical pattern of movement was seen in 3 of the normal subjects. In all 3 this was localised to the apex, extended 19 to $40 \mathrm{~mm}$, and was associated with abnormal inward movement during isovolumic relaxation of 4 to $8 \mathrm{~mm}$.

Type II (9 patients) (Figs. 7 to 9 )

In this group, the overall amplitude of movement in the affected area was by definition less than 60 per cent of that in the control regions, having a mean amplitude of $4 \pm 2 \mathrm{~mm}$. In 3 patients there 
DISTRIBUTION OF ISOVOLUMIC CONTRACTION ABNORMALITIES

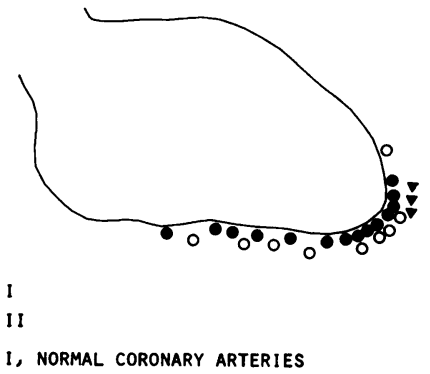

Fig. 6 Positions of the plots showing greatest amplitude of outward movement before aortic valve opening in 26 patients with type I or type II abnormalities.

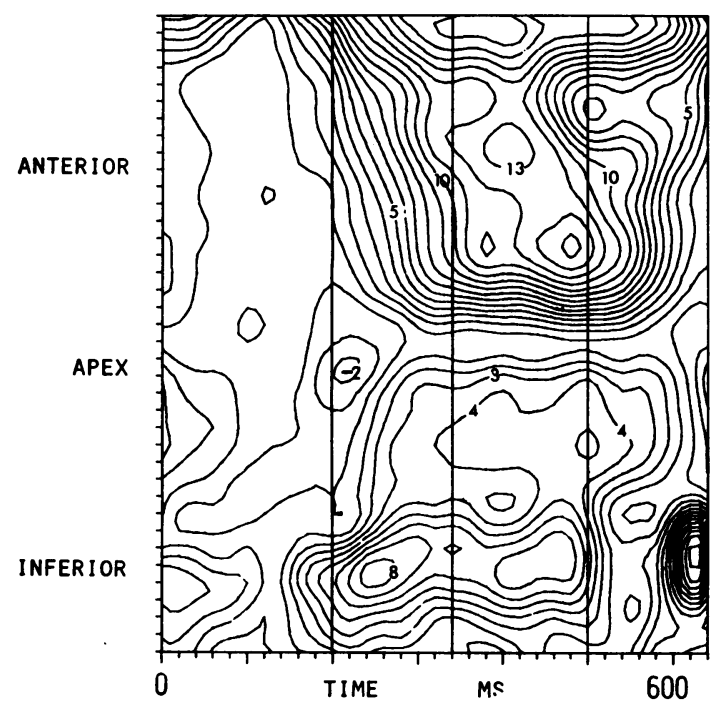

Fig. 7 Contour plot of the left ventriculogram of a patient with a type II abnormality.

was a region of outward movement throughout ejection. The time of maximum outward displacement varied widely with respect to aortic valve opening from 0 to $160 \mathrm{~ms}$, and the characteristic bifid form of the velocity trace was not seen. Peak velocities of inward movement were also significantly less than in patients included in the previous group $(3 \pm 1$ $\mathrm{cm} / \mathrm{s}, \mathrm{P}<0.01$ ) as well as in control regions of the same ventricle $(9 \pm 3 \mathrm{~cm} / \mathrm{s}, P<0.01)$. The distribution (Fig. 6) and extent (22 to $47 \mathrm{~mm}$ ) were similar, but only one showed inward movement of endocardium during isovolumic relaxation, and that by $2 \mathrm{~mm}$.

Type III (21 patients) (Fig. 10)

A third type of abnormality was recognisable, where there was no outward movement, but instead premature inward movement of the endocardium along the inferior wall by more than $2 \mathrm{~mm}$ before aortic valve opening. In 10 of these patients, it was associated with a type I abnormality, usually at the apex, thus constituting another manifestation of the change in cavity shape occurring in isovolumic contraction. In the remainder, it was the only abnormality detected.

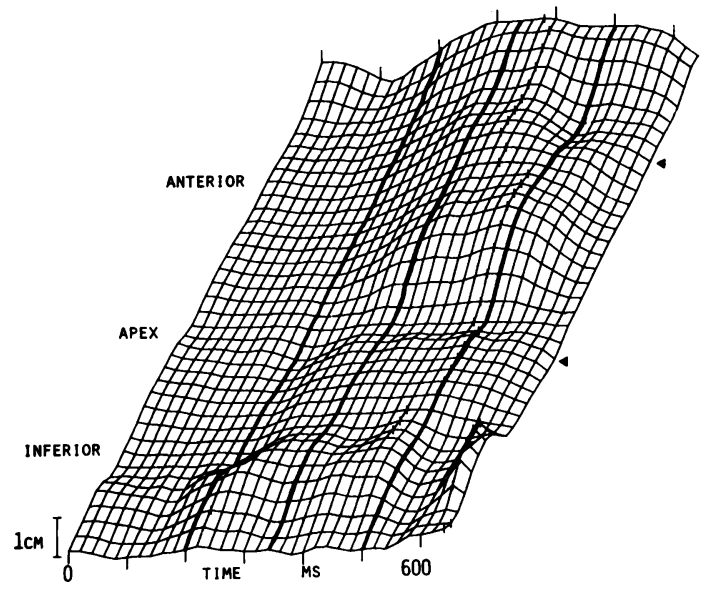

Fig. 8 Isometric plot of the data of Fig. 7. The plots indicated by arrows are analysed in greater detail in Fig. 9.

\section{CLINICAL ASSOCIATIONS (Table)}

(a) Amplitude of wall movement

The mean ejection fraction in patients with type I or type III abnormalities was $70 \pm 12$ per cent and $70 \pm 16$ per cent, respectively, significantly greater than the mean value for the whole group (60 \pm $17 \%, \mathrm{P}<0.01$ ), while that in patients with type II abnormalities was less $(53 \pm 16 \%, P<0.01)$. This pattern was reflected in the increased incidence of regional reduction in the amplitude of wall movement in type II as compared with the other two $(P<0.05)$ regardless of whether this occurred at the site of abnormal function during isovolumic contraction.

(b) Coronary artery disease

There was no significant difference in the incidence or distribution of coronary artery disease in the 
Table Clinical associations

\begin{tabular}{|c|c|c|c|c|}
\hline & \multicolumn{3}{|c|}{ Isovolumic contraction abnormal } & \multirow{2}{*}{$\begin{array}{l}\text { Isovolumic } \\
\text { contraction } \\
\text { normal }\end{array}$} \\
\hline & Type I & Type II & Type III† & \\
\hline \multicolumn{5}{|l|}{$\begin{array}{l}\text { Wall movement } \\
\text { amplitude }\end{array}$} \\
\hline Normal & 9 & 1 & 7 & 30 \\
\hline $\begin{array}{l}\text { Anterior } \\
\text { hypokinesis }\end{array}$ & 0 & 0 & 1 & 7 \\
\hline Inferior & & & & \\
\hline hypokinesis & 6 & 5 & 3 & 12 \\
\hline Apical hypokinesis & 2 & 1 & 0 & 7 \\
\hline Generalised & 0 & 2 & 0 & 12 \\
\hline \multicolumn{5}{|l|}{ Coronary artery } \\
\hline $\mathrm{LAD}^{\star}$ & 8 & 2 & 2 & 14 \\
\hline $\mathrm{RCA}^{\star}$ & 0 & 1 & 1 & 7 \\
\hline LAD + RCA & 4 & 2 & 2 & 9 \\
\hline 3 vessel & 2 & 3 & 6 & 23 \\
\hline Normal & 3 & 0 & $\mathbf{0}$ & 12 \\
\hline Not known & 0 & 1 & $\mathbf{0}$ & 3 \\
\hline \multicolumn{5}{|l|}{ Electrocardiogram } \\
\hline No $Q$ waves & 10 & 2 & 7 & 45 \\
\hline Anterior $Q$ & 3 & 7 & 1 & 10 \\
\hline Inferior $Q$ & 3 & 0 & 3 & 12 \\
\hline Anterior $+\inf Q$ & 0 & 0 & $\mathbf{0}$ & 1 \\
\hline LBBB & 1 & 0 & 0 & 0 \\
\hline
\end{tabular}

LAD, left anterior descending coronary artery disease; RCA, right coronary artery disease; LBBB, left bundle-branch block.

$\star \pm$ Additional circumflex disease.

†Patients with type III abnormality only, this column does not include those with additional type I.

three groups, either in comparison with one another, or with the remainder of the group in whom isovolumic contraction was normal.

\section{(c) Electrocardiogram}

The incidence of $Q$ waves was abnormally high in patients with outward endocardial movement before aortic valve opening (types I and II, $P<0.025$, $\chi^{2}=8 \cdot 5$ ). This was because of the high incidence of anterior $Q$ waves in these patients. Though the figures were small, and therefore not statistically significant, this increased incidence of anterior $Q$ waves occurred almost exclusively in patients with type II abnormalities. By contrast, the incidence of inferior $Q$ waves throughout all three groups in whom isovolumic contraction was abnormal was not distinguishable from that predicted by chance.

\section{(d) Isovolumic relaxation}

Abnormal inward movement during isovolumic relaxation was present in $\mathbf{4 1}$ patients: in 15 of these, there were abnormalities of isovolumic contraction involving the same segment. This distribution differs significantly from chance $\left(\chi^{2}=8.5, \mathrm{P}<\right.$ 0.01 ); in all cases but 1 , these abnormalities of isovolumic contraction were of type $I$.
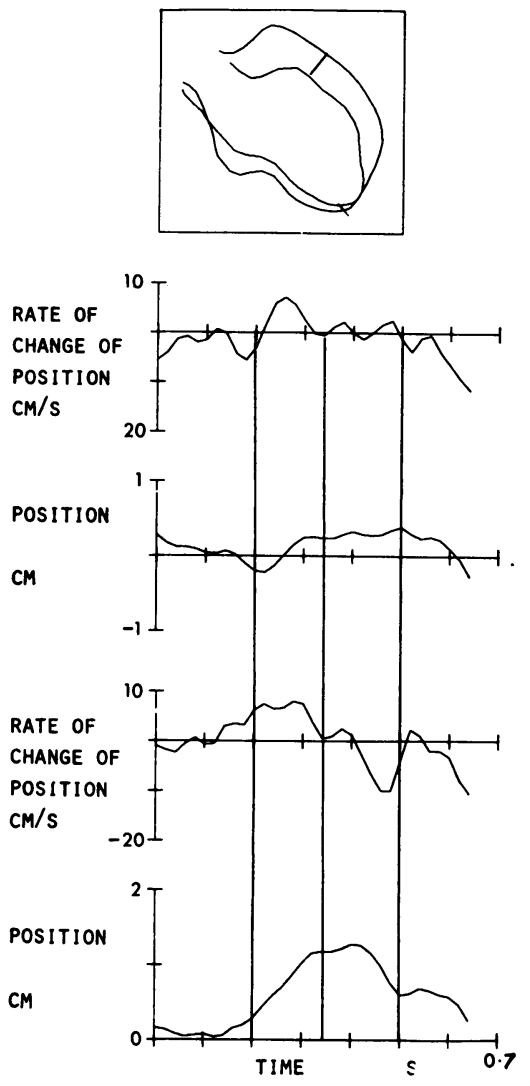

Fig. 9 Analysis of regional wall movement in a patient with a type II abnormality. Layout as Fig. 5. There is outward movement before and immediately after aortic valve opening, but little inward movement during ejection or isovolumic relaxation.

\section{Discussion}

The change in the myocardium from the relaxed to the contracted state that occurs during isovolumic contraction is accompanied by only minor alterations in cavity configuration, suggesting that it occurs almost synchronously throughout the left ventricle. Our results in normal subjects agree closely with those reported in the conscious dog (Rankin et al., 1976) in showing that these consist of a small reduction in transverse dimension, no change in long axis, and an alteration in cavity shape towards a less spherical configuration. A small reduction in transverse dimension has also been recorded in man by angiography (Karliner et al., 1971), and by echocardiography (Venco et al., 1977). This reduction appeared to be caused by inward move- 


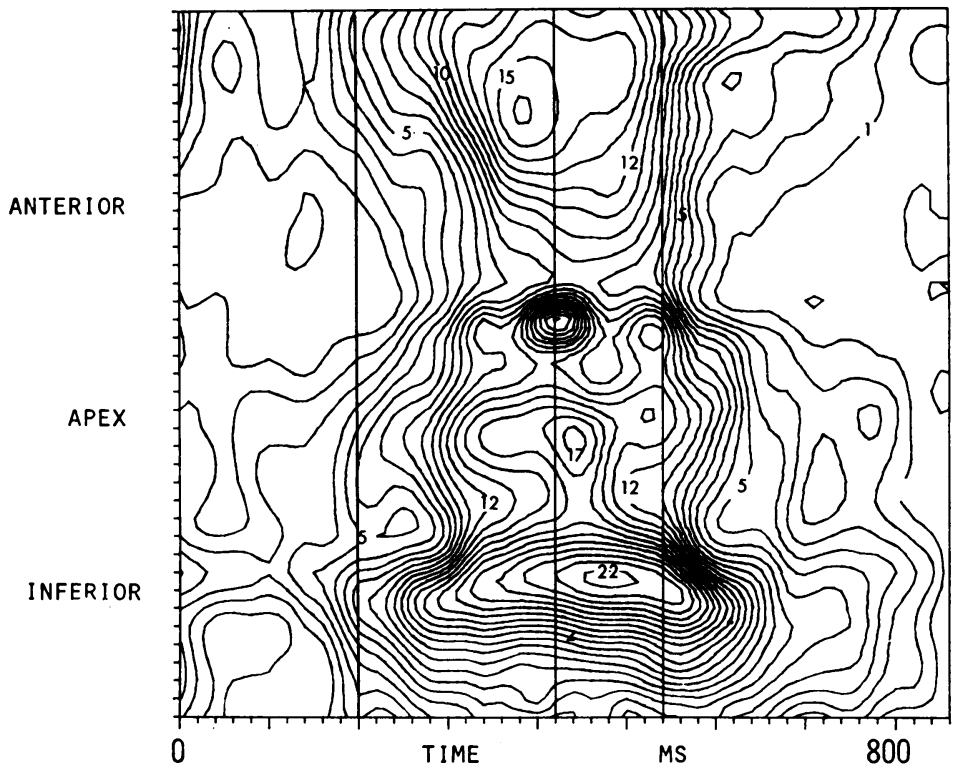

Fig. 10 Contour plot of the left ventriculogram of a patient with a type III abnormality, showing premature inward movement in the inferior region of the cavity. ment of the upper part of the anterior wall, adjoining the aortic root, rather than by symmetrical change around the whole cavity outline.

Angiocardiography appears to be a satisfactory means of studying wall movement at this time in the cardiac cycle, in that endocardial position corresponds closely with the outer border of angiographic dye. The method is not, therefore, open to those errors that arise in late systole and early diastole as a result of endothelial infolding (Hugenholtz et al., 1969). However, in any region, endocardial movement can only be assessed relative to some arbitrary reference point, so that such measurements are determined by the combined effects of local movement and overall displacement of the heart within the thorax. For this reason, we prefer to regard the characteristic sequence of endocardial movement occurring in normal subjects merely as a consistent pattern, dependent on the method of display used, rather than as indicating the order of activation or the onset of tension development. Nevertheless, its uniformity made it a useful basis for comparison with that seen in patients with coronary artery disease.

The commonest disturbance of this characteristic pattern was a discrete area of outward movement involving 2 to $4 \mathrm{~cm}$ of the circumference of the cavity in the projection used (type I). Though these disturbances were present at the time of aortic valve opening, they reached their greatest extent 50 to $100 \mathrm{~ms}$ later, and were associated with a significant increase in local wall curvature. This combination of outward movement and increased curvature would be predicted if the affected area were unable to support stress, suggesting that the onset of tension development was locally delayed (Herman and Gorlin, 1969). Subsequent inward movement, however, showed the development of contractile activity later in systole, and its persistence into the period of isovolumic relaxation suggests that the whole process of contraction was delayed relative to normal areas. With amplitude and duration of inward movement not differing significantly from normal regions in the same ventricle, local myocardial function did not appear to be significantly impaired. Though peak rates of inward endocardial movement were also normal, they were characteristically recorded on two occasions-early and late in systole. The early peak corresponded to inward movement of the affected area towards its position at end-diastole at a time when local curvature was still increased, so that local wall stress was lower than in a normal region at the same cavity pressure. The second peak corresponded to inward wall movement during isovolumic relaxation when pressure was falling. On both occasions, therefore, peak rates of wall movement were recorded at times of reduced local wall stress.

These abnormalities of endocardial movement appear similar to those described in previous studies. Leighton et al. (1975) found abnormally contracting segments at mid-ejection in 27 out of 42 patients with coronary artery disease. Though 
these authors specifically excluded wall movement before aortic valve opening from consideration, it seems likely that regions with properties similar to those of type I were being studied. More recently, the same group have documented related abnormalities in patients with normal coronary arteries (Altieri et al., 1976). The presence of segmental contraction delay may also be responsible for the reduced early systolic ejection rate seen in patients with coronary artery disease by Johnson et al. (1975). All these results are compatible with the existence of abnormal regions in which the onset of contraction is delayed though its subsequent course approximates to normal. The coincidence of normal peak rates of inward movement only with times of reduced wall stress, however, raised the possibility of additional left ventricular disease.

In regions showing type II behaviour, the contribution of local impairment of myocardial function was much clearer. In 3 cases there was aneurysmal behaviour, and in the remainder, the amplitude, peak rate, and duration of inward movement were all reduced, with inward movement occurring during isovolumic relaxation in only one. The significance of premature inward movement of endocardium in the inferior part of the cavity (type III) is harder to assess. The normal amplitude velocity, and duration of inward movement all suggest that it is not a sign of local disease. Its frequent association with type I abnormalities suggests that it may be a compensatory phenomenon in a period of constant ventricular volume, inward movement in one part of the cavity being, of necessity, associated with outward movement elsewhere.

The occurrence of similar abnormalities in 3 patients in whom coronary artery anatomy was normal may shed doubt on their pathological significance, raising the possibility that they may result from some other factor, such as overall movement of the heart within the thorax. This seems unlikely, since a local increase in curvature cannot be explained in this way, but requires the presence of some local disturbance. In addition, a previous study (Doran et al., 1978), has shown close correlation between angiographic detection of abnormal endocardial movement before aortic valve opening and dimension changes during the period of isovolumic contraction detected by echocardiography. It is not possible to study truly normal subjects by angiography in the same way as by echo, and the latter technique has shown that in such individuals, wall motion disturbances do not occur during isovolumic contraction. The angiographic findings thus confirm that wall movement abnormalities may occur in patients with angina and normal coronary arteries, and indicate that they are abnormalities of timing, rather than of amplitude or direction of wall movement, which are more commonly recognised.

Endocardial movement during isovolumic contraction has functional significance. An increase in dimension as pressure rises leads to dissipation of energy, with a reduction in the efficiency of energy transfer from the myocardium to the circulation (Gibson and Brown, 1976). The rate of rise of pressure is reduced with delay in aortic valve opening and reduction in ejection time. This may affect 'isovolumic indices' of left ventricular performance such as $\mathrm{dP} / \mathrm{dt} / \mathrm{P}$ or the pre-ejection period, which therefore should not be used to give information about overall left ventricular function or the rate of tension development. In addition, the effects of delayed onset of contraction appear to persist throughout ejection and are still apparent during isovolumic relaxation, causing inward movement of endocardium before mitral valve opening. The significance of such changes has been described elsewhere (Gibson et al., 1976); in the present series of patients, over one-third of relaxation abnormalities occurred in segments which had been the site of delayed onset of contraction.

This study raises a number of further questions. The distribution of lesions around the apex and adjoining area of the inferior wall was unexpected and has no obvious basis in myocardial architecture or in the distribution of either conducting system or blood supply. Indirect confirmation of this asymmetrical localisation, however, comes from echocardiographic studies which suggest the relative rarity of outward wall movement at the base of the cavity when isovolumic contraction abnormalities are known to be present (Doran et al., 1978). Those of type II occurred more frequently in ventricles with other evidence of myocardial disease. In spite of their inferior position, they were frequently associated with anterior $Q$ waves on the electrocardiogram, though not with anterior hypokinesis. This raises the possibility that the primary disturbance was in activation, and indicates that such effects may occur in regions of the ventricle distant from those usually thought to be involved in anterior infarction. There is no evidence at all as to the mode of production of type I abnormalities. In this population of patients with angina, they were unrelated to the electrocardiogram, to regional abnormalities of overall amplitude, and even to the presence or absence of coronary artery disease, regardless of its distribution. In these circumstances, some regional abnormality of activation seems more likely than a disturbance of the contractile process itself, but we have no direct evidence of its presence, 
or conception of its nature. Finally, the absence of wall movement disturbances in isovolumic periods in truly normal subjects as shown by echocardiography suggests that when present these abnormal movements may be related to a predisposition to develop angina, though we can only conjecture on the mechanism by which they do so.

Comparison of events during isovolumic contraction and relaxation indicated the close relation between the two, not only because both may occur in the same region of ventricle, but because the disturbances that they cause are so similar. Maintenance of constant left ventricular dimensions during the two isovolumic periods when the myocardium is rapidly changing state presupposes a high degree of organisation. This may not be obvious in normal subjects, but its absence becomes obtrusive in patients with heart disease. The functional significance of this loss is the same in both isovolumic periods: impaired mechanical efficiency, inappropriate wall position during periods of rapid volume change, and an increase in the 'lost' time in the cardiac cycle, when the ventricle is neither ejecting nor filling, and in addition both may be associated with disturbed function of otherwise normal regions. It is clear, therefore, that these abnormalities may cause impairment of overall left ventricular performance in a way which cannot be predicted from simple tests of muscle function, and to a degree out of proportion to their actual extent. Their existence stresses the significance of the high degree of organisation implicit in the maintenance of normal left ventricular function, and their detection in individual patients gives some indication of its loss.

The computing equipment used in this study was supplied by the DHSS as part of its experimental programme.

\section{References}

Altieri, P. I., Pollack, M. E., and Leighton, R. F. (1976).
Hypokinetic and tardokinetic segments in patients with angina-like pain and angiographically normal coronary arteries. In Proceedings of 7 th European Congress of Cardiology, p. 251.

Doran, J. H., Traill, T. A., Brown, D. J., and Gibson, D. G. (1978). Detection of abnormal left ventricular wall movement during isovolumic contraction and early relaxation. Comparison of echo- and angiocardiography. British Heart fournal, 40, 367-371.

Gibson, D. G., and Brown, D. J. (1975). Continuous assessment of left ventricular shape in man. British Heart fournal, 37, 904-910.

Gibson, D. G., and Brown, D. J. (1976). Assessment of left ventricular systolic function from simultaneous echocardiographic and pressure measurements. British Heart fournal, 38, 8-17.

Gibson, D. G., Prewitt, T. A., and Brown, D. I. (1976). Analysis of left ventricular wall movement during isovolumic relaxation in man and its relation to coronary artery disease. British Heart fournal, 38, 1010-1019.

Herman, M. V., and Gorlin, R. (1969). Implications of left ventricular asynergy. American fournal of Cardiology, 23, 538-547.

Hugenholtz, P. G., Kaplan, E., and Hull, E. (1969). Determination of left ventricular wall thickness by angiocardiography. American Heart fournal, 78, 513-522.

Johnson, L. L., Ellis, K., Schmidt, D., Weiss, M. B., and Cannon, P. J. (1975). Volume ejected in early systole. A sensitive index of left ventricular performance in coronary artery disease. Circulation, 52, 378-389.

Karliner, J. S., Bouchard, R. J., and Gault, J. H. (1971). Dimensional changes of the human left ventricle prior to aortic valve opening. Circulation, 44, 312-322.

Leighton, R. F., Pollack, M. E. M., and Welch, T. G. (1975). Abnormal left ventricular wall motion at mid-ejection in patients with coronary heart disease. Circulation, 52, 238-244.

McDonald, I. G. (1970). The shape and movements of the human left ventricle during systole. American fournal of Cardiology, 26, 221-230.

Rankin, I. S., McHale, P. A., Arentzen, C. E., Ling, D., Greenfield, J. C., Jr., and Anderson, R. W. (1976). The three dimensional dynamic geometry of the left ventricle in the conscious dog. Circulation Research, 39, 304-313.

Venco, A., Gibson, D. G., and Brown, D. J. (1977). Relation between apex cardiogram and changes in left ventricular pressure and dimension. British Heart fournal, 39, 117-125.

Requests for reprints to Dr Derek Gibson, Cardiac Department, Brompton Hospital, Fulham Road, London SW3 6HP. 